\title{
Rastreamento da função cognitiva de idosos não-institucionalizados
}

\author{
Sreering cogitivefuntion of noninstittiondized ddely
}

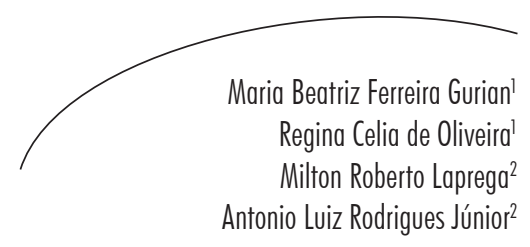

\section{Resumo}

O objetivo deste trabalho foi avaliar a função cognitiva dos idosos e determinar a frequência do déficit cognitivo, estratificando-os por idade, escolaridade, passatempo, relação social, doenças crônicas informadas e depressão. A uma amostra aleatória de 394 idosos com idade igual ou maior que 60 anos do município de Batatais (SP) foi aplicado questionário sobre condições socioeconômicas, saúde e Escala de Depressão Geriátrica (EDG). Para rastrear o déficit cognitivo, foi utilizado o MEEM - Mini-Exame do Estado Mental modificado. Na avaliação do desempenho cognitivo, usou-se ponto de corte 23 . Observou-se que $81,7 \%$ dos idosos ficaram acima desse ponto e 18,3\% ficaram abaixo. Os idosos que tiveram os escores mais elevados foram associados a fatores como idade (60-69 anos), nível de escolaridade, hábito de leitura, boa relação social, principalmente com familiares, e não ter hipertensão arterial, diabete, incontinência urinária, catarata e ou sintomas depressivos. O desempenho cognitivo global dos idosos avaliado pelo instrumento baseado no MEEM revelou que aqueles com escores abaixo do ponto de corte tiveram proporção semelhante à encontrada em outros estudos.

\section{Abstract}

This study aimed to evaluate the cognitive function of elderly people and determine the frequency of cognitive impairment, stratified by age, education, hobby, social relationship, informed chronic diseases and depression. In a random sample of 394 elderly aged over 60 years in the city of Batatais, state of São Paulo, Brazil, was administered a questionnaire on socioeconomic conditions, health and Geriatric

Key words: Aged. Cognition Disorders. Evaluation.

Screening.

\footnotetext{
Centro Universitário Claretiano, Curso de Fisioterapia. Batatais, SP, Brasil

2 Faculdade de Medicina de Ribeirão Preto, Departamento de Medicina Social. Universidade de São Paulo. Ribeirão Preto, SP, Brasil.
}

Pesquisa financiada pela Fundação de Amparo e Pesquisa do Estado de São Paulo (FAPESP), baseado em dissertação de Mestrado apresentada a Faculdade de Medicina Social de Ribeirão Preto da Universidade de São Paulo.

Correspondência / Correspondence

Maria Beatriz Ferreira Gurian

Centro Universitário Claretiano

Rua Dom Bosco, 466

14300-000 - Batatais, SP, Brasil

E-mail: bia@claretiano.edu.br

Palavras-chave: idoso. Transtornos Cognitivos. Avaliação. Rastreamento. 
Depression Scale (GDS). To track the cognitive impairment, we used the MMSE Mini Mental State Examination modified. In the assessment of cognitive performance, we used a cutoff point of 23 . It was observed that $81.7 \%$ of the subjects were above this point and $18.3 \%$ were below. The elderly who had higher scores were associated with factors such as age (60-69 years), educational level, reading habits, good social relations, especially with family and no hypertension, diabetes, urinary incontinence, cataracts and/or depressive symptoms. The global cognitive performance of older people assessed by the tool based on the MMSE showed that those with socres below the cutoff point had a similar proportion to that found in other studies.

\section{INTRODUÇÃO}

O crescimento da população de idosos, em números absolutos e relativos, é um fenômeno mundial que decorre da melhoria das condições de vida e assistência à saúde. Essa alteração do perfil demográfico tem como consequências o aumento da prevalência de doenças crônicodegenerativas e a modificação do perfil epidemiológico de morbi-mortalidade das comunidades, acarretando uso desproporcional dos serviços de saúde ${ }^{1}$ pelos idosos, trazendo implicações sociais e econômicas à saúde pública. ${ }^{2}$

Sabe-se que tanto as doenças físicas como as mentais podem levar à perda da independência e autonomia, sendo fatores de risco importantes para a mortalidade. Doenças como depressão e demência já estão, em todo o mundo, entre as principais causas de anos vividos com incapacidade funcional.

Durante o processo de envelhecimento, $15 \%$ das pessoas desenvolvem inicialmente incapacidade cognitiva progressiva. Desse total, mais ou menos $5 \%$ das pessoas acima de 65 anos e $20 \%$ acima de 80 desenvolvem demência de grau moderado a grave. ${ }^{3}$

Ramos \& Macedo $^{4}$ relataram que perdas cognitivas, juntamente com o comprometimento da memória, estão entre os problemas mais comuns trazidos pelo idoso durante a consulta médica. Resultados do estudo de Geerlings et $a .^{5}$ sugerem que o déficit cognitivo precede a demência entre idosos. Bickel \& Cooper ${ }^{6}$ relataram que identificar o comprometimento cognitivo leve, no rastreamento, pode ser útil na avaliação do risco para demência dois a três anos antes de a condição se tornar clinicamente aparente. Esses quadros são frequentes e passam despercebidos, havendo necessidade de distinguir entre manifestações iniciais de doença e modificações associadas ao processo normal de envelhecimento. Informações sobre o tema na literatura brasileira são poucas, e a estimativa é de 5 a 30\% de idosos com quadro demencial. ${ }^{7}$

O presente estudo teve como objetivo avaliar a função cognitiva de idosos não institucionalizados, verificando seu desempenho cognitivo, além de relacioná-lo com idade, escolaridade, passatempos, relação social, doenças crônicas informadas e depressão, com o propósito de, conhecendo a realidade desses idosos no município nesses quesitos, fornecer subsídios para a elaboração de medidas de controle e prevenção.

\section{METODOLOGIA}

O estudo foi realizado na região urbana do município de Batatais, no nordeste do Estado de São Paulo.

Segundo o censo de 2000, ${ }^{8}$ existiam 15,5 milhões de brasileiros com mais de 60 anos no Brasil. No município de Batatais, havia 5.755 idosos, que representavam em torno de $8.8 \%$ da população. 
A amostra foi selecionada aleatoriamente através de listas de idosos fornecidas pela Secretaria Municipal da Saúde, as quais continham a relação de moradores com idade igual ou maior que 60 anos, cadastrados pelos agentes comunitários de saúde em todos os bairros da zona urbana do município. Foram fornecidas sete listas, cada uma representando uma área envolvendo seus respectivos bairros, de acordo com a divisão da Secretaria da Saúde e, nestas, os idosos eram classificados por rua e número de moradia. O total de cadastrados foi de 4.414 , o que representa $75,5 \%$ da população estimada dessa faixa etária no município.

Utilizando-se a estimativa de 15\% para a prevalência de déficit cognitivo em idosos, para um nível de significância de 95\%, ${ }^{9}$ obteve-se o valor de 394 para o tamanho da amostra. Esses idosos foram submetidos à pesquisa domiciliar realizada entre março e junho de 2002, sendo adotados os seguintes critérios de exclusão: residir em instituições, ausência no terceiro contato, doença grave e recusa.

Participaram dez entrevistadores do terceiro ano do curso de Fisioterapia do Centro Universitário Claretiano de Batatais devidamente treinados para esse fim. Foram usados os seguintes instrumentos:

Questionário contendo 29 questões referentes às condições socioeconômicas e de saúde, obtidas com o idoso, familiar e/ou acompanhantes.

Escala de Depressão Geriátrica (EDG) é um instrumento utilizado para detecção de depressão no idoso. ${ }^{10}$ Foi usada a versão reduzida com 15 questões, a qual vem sendo utilizada frequentemente em razão de o tempo gasto com sua aplicação ser substancialmente curto. $O$ ponto de corte empregado foi $5 .^{10}$

Como instrumento para o rastreamento do déficit cognitivo, foi utilizado o MEEM Mini-Exame do Estado Mental, ${ }^{11}$ modificado por Seabra et al. ${ }^{12} \mathrm{O}$ ponto de corte utilizado foi 23, como na maior parte dos trabalhos internacionais.

A análise estatística utilizou a medida MEEM dicotomizada (acima e abaixo do ponto de corte), para a avaliação da função cognitiva. Tendo sido definida uma variável binária, empregouse o modelo de regressão logística para avaliar os dados do estudo, ${ }^{13}$ usando o pacote STATA ${ }^{\circledR}$ nesta avaliação estatística.

O estudo foi aprovado pelo Comitê de Ética em Pesquisa do Centro de Saúde Escola da Faculdade de Medicina de Ribeirão Preto sob o número 0012/CEP-CSE-FMRP-USP e todos os participantes assinaram o termo de consentimento livre e esclarecido.

\section{RESULTADOS}

Foram avaliados 394 idosos. Destes, 322 $(81,7 \%)$ obtiveram escore acima de 23 pontos e $72(18,3 \%)$ obtiveram escore igual e abaixo de 23 pontos.

Observaram-se, dentre os idosos com MEEM $>$ 23, 198 idosos na faixa etária de 60-69 anos, 94 na faixa etária 70-79 anos e 34 com idade maior que 80 anos, e dentre os com MEEM $\leq 23,20$ idosos na faixa etária 60-79 anos, 32 na faixa etária 70-79 anos e 16 com idade maior que 80 anos (tabela 1). 
Tabela 1 - Distribuição da frequência dos escores do instrumento baseado no MEEM e variável faixa etária, escolaridade, leitura, relação social/parentes, doenças crônicas, EDG. Batatais, São Paulo, 2002. $(\mathrm{N}=394)$

\begin{tabular}{|c|c|c|}
\hline \multirow{2}{*}{$\begin{array}{l}\text { Variáveis } \\
\text { Faixa etária }\end{array}$} & \multicolumn{2}{|c|}{ MEEM } \\
\hline & $>23$ & $\leq 23$ \\
\hline \multicolumn{2}{|l|}{ Faixa etária } & 20 \\
\hline $70-79$ & 94 & 32 \\
\hline$>80$ & 32 & 16 \\
\hline \multicolumn{3}{|l|}{ Escolaridade } \\
\hline Nunca frequentou & 59 & 46 \\
\hline 1-4 anos & 201 & 19 \\
\hline+5 anos & 66 & 3 \\
\hline \multicolumn{3}{|l|}{ Passatempo / leitura } \\
\hline Muito frequente/frequente/as vezes/rara & 233 & 18 \\
\hline Nunca & 93 & 50 \\
\hline \multicolumn{3}{|l|}{ Relação social / parentes } \\
\hline Diariamente/Semana/Mensal & 315 & 61 \\
\hline $1 \mathrm{x} /$ ano & 13 & 4 \\
\hline \multicolumn{3}{|l|}{ Doenças crônicas } \\
\hline Hipertensão arterial & 168 & 44 \\
\hline Diabete & 39 & 15 \\
\hline Catarata & 55 & 27 \\
\hline Incontinência & 22 & 14 \\
\hline \multicolumn{3}{|l|}{ Escala de Depressão Geriátrica (EDG) } \\
\hline$\leq 5$ & 264 & 45 \\
\hline$>5$ & 62 & 23 \\
\hline
\end{tabular}


O modelo de regressão logística mostrou associação significativa do desempenho cognitivo no que se refere à faixa etária de 70 79 anos $(\mathrm{OR}=2,3 ; \mathrm{p}=0,022)$ e na faixa etária com mais de 80 anos $(\mathrm{OR}=2,3 ; \mathrm{p}=0,006)$ (tabela 2).

Tabela 2 - Correlação do desempenho cognitivo através do instrumento baseado no MEEM com as variáveis estudadas. Batatais, São Paulo, 2002. (N=394)

\begin{tabular}{lccc}
\hline Variáveis & $f$ MEEM $\leq 23$ & OR & $\mathrm{P}<0,05$ \\
\hline Faixa etária & 32 & 2,3 & 0,022 \\
$70-79$ & 16 & 2,3 & 0,006 \\
$>80$ & & & \\
Escolaridade & 19 & 0,12 & 0,0001 \\
$1-4$ & 3 & 0,07 & 0,001 \\
$5-8$ & & & \\
Passatempo (leitura) & 50 & 7,9 & 0,001 \\
Nunca lê & & & 0,041 \\
Relação social (parentes) & 4 & 7,2 & \\
Não se relaciona & & & 0,056 \\
Problemas de saúde & 44 & 1,8 & 0,042 \\
Hipertensão & 15 & 2,1 & 0,001 \\
Diabete & 14 & 3,7 & 0,0001 \\
Incontinência urinária & 27 & 3,3 & 0,008 \\
Catarata & & & \\
Escada de Depressão Geriátrica (EDG) & 23 & 2,2 & \\
$>5$ & & & \\
\hline
\end{tabular}


Foi observado entre os idosos com MEEM $>$ 23, que: 59 nunca frequentaram escola, 201 tiveram de um a quatro anos de escolaridade e 66 cursaram mais que cinco anos. Entre aqueles com MEEM $\leq$ 23: 46 nunca frequentaram escola, 19 tiveram de um a quatro anos de escolaridade e três frequentaram mais que cinco anos (tabela 1). Estes resultados são significativos: $\mathrm{OR}=0.12 \mathrm{e}$ $\mathrm{p}<0,0001$ para os indivíduos com um a quatro anos de escolaridade e OR $=0.07$ e $\mathrm{p}<0,0001$ para escolaridade entre cinco e oito anos (tabela 2).

Dos idosos que obtiveram resultados com o MEEM > 23, 59 liam com muita frequência; 33, frequentemente; 86 , às vezes; 55 , raramente e 93 nunca liam. Entre aqueles com o MEEM $\leq 23$, três idosos liam com muita frequência; quatro, frequentemente; cinco, às vezes; seis, raramente e 50 idosos nunca liam (tabela 1). Na avaliação da influência dos passatempos, quando relacionados ao desempenho cognitivo, houve associação significativa quanto à ausência do hábito de leitura (nunca leem) $(\mathrm{OR}=7,9 ; \mathrm{p}=0,001)$, quando comparados com os indivíduos que leem com alguma frequência (muito frequente, frequente, às vezes e raramente) (tabela 2).

Observou-se que entre os idosos com o MEEM $>$ 23, 152 relacionavam-se diariamente com parentes; 112, semanalmente; 47, mensalmente; 13 , uma vez por ano e dois idosos não souberam informar (tabela 1). Entre aqueles com o MEEM $\leq$ 23, 24 relacionavam-se diariamente com parentes; 24 , semanalmente; 13 , mensalmente; quatro, uma vez por ano e três idosos não souberam informar. Verificou-se significância quando comparados os indivíduos que não se relacionam com os familiares com aqueles que se relacionam diariamente $(\mathrm{OR}=$ 7.2; $\mathrm{p}=0,041)$ (tabela 2).

Foram observados, dentre os idosos com MEEM > 23, 158 casos sem e $168 \mathrm{com}$ hipertensão, e dentre os com MEEM $\leq 23,24$ sem e 44 com hipertensão; dentre os idosos com MEEM $>23,287$ casos sem e 39 com diabetes, e dentre os com MEEM $\leq 23,53 \mathrm{sem}$ e $15 \mathrm{com}$ diabetes; dentre os idosos com MEEM > 23, 304 casos sem e 22 com incontinência urinária, dentre os com MEEM $\leq 23,54 \mathrm{sem}$ e $14 \mathrm{com}$ incontinência urinária; dentre os idosos com
MEEM > 23, 271 casos sem e 55 com catarata, dentre os com MEEM $\leq 23,41$ sem e $27 \mathrm{com}$ catarata (tabela 1). O estudo da associação entre os problemas de saúde relatados e os estratos do desempenho cognitivo mostrou que houve associação significativa entre a ocorrência de hipertensão $(\mathrm{OR}=1,8 ; \mathrm{p}=0,056)$, de diabete $(\mathrm{OR}=2,1 ; \mathrm{p}=0,042)$, de incontinência urinária $(\mathrm{OR}=3,7 ; \mathrm{p}=0,001)$ e de catarata $(\mathrm{OR}=3,3 ; \mathrm{p}$ $<0,0001$ ) (tabela 2).

Os dados relacionados à Escala de Depressão Geriátrica (EDG) e MEEM estão descritos na tabela 1, e a análise estatística revelou que existe significância entre essas duas variáveis $(\mathrm{OR}=$ $2,2 ; \mathrm{p}=0,008)$ (tabela 2).

\section{DISCUSSÃO}

No presente estudo, a frequência do desempenho cognitivo dos que obtiveram escore igual e abaixo de 23 pontos foi de 18,3\%. Em comparação com outros estudos, nossos resultados revelaram proporção inferior. No estudo de Bennet et al., ${ }^{14}$ realizado em uma ordem católica dos Estados Unidos, os resultados mostraram que $26,4 \%$ tinham déficit cognitivo. Fichter et al. ${ }^{15}$ realizaram estudo com idosos em comunidade com 65 anos ou mais e relataram prevalência de $46,5 \%$. O estudo de Silberman et al., ${ }^{16}$ com idosos vivendo em comunidade com 60 anos ou mais, que recebiam assistência de saúde e educação do Ambulatório do Serviço de Saúde Comunitária do Hospital da Cidade de Porto Alegre, RS, encontraram frequência de $29 \%$. E Machado et al. ${ }^{17}$ encontraram frequência de $36,5 \%$ entre idosos com 60 anos ou mais, inscritos em um programa para a terceira idade no município de Viçosa, MG.

Os resultados observados confirmaram que o escore do instrumento baseado no MEEM se relaciona de forma significativa com a idade e com o nível educacional do idoso; nesse caso, é necessário levar em consideração que a variável educação é complexa e individual, pois a capacidade cerebral de um indivíduo é determinada, em grande parte, pelo desenvolvimento do SNC na vida fetal e durante os primeiros anos de vida. Nessas fases, o cérebro está mais suscetível à influência de riscos 
ambientais mais comumente associados ao baixo nível educacional, como nutrição inadequada, escassa estimulação neuropsicomotora, maior exposição a condições precárias de vida, podendo refletir na vida adulta e contribuir para o declínio intelectual no idoso. ${ }^{18}$ Em outras palavras, quanto mais jovem e maior o nível de escolaridade, maior o escore do instrumento baseado no MEEM. ${ }^{10,19-28}$

Em relação ao hábito de leitura, os resultados podem ter mostrado significância por estarem associados com o número de analfabetos da amostra, sendo necessário, portanto, tomar cuidado ao se tentar estabelecer uma relação de causa-efeito entre essas variáveis. Dados do estudo de Germano Neto ${ }^{23}$ mostraram que idosos que não tinham o hábito de ler tiveram uma média no MEEM de 23,47 e os que liam habitualmente obtiveram média de 27,05. O autor argumentou que indivíduos mais dotados intelectualmente teriam tendência a adotar hábitos compatíveis com sua capacidade intelectual, ou seja, leem mais porque têm um intelecto mais desenvolvido, sendo o inverso também verdadeiro.

O que se pode afirmar é que os efeitos da educação incluem a aprendizagem de conhecimentos e habilidades específicas, mas também aumentam a eficiência geral no processamento e no manejo de informação.? Bertolucci et al. ${ }^{27}$ relatam que, provavelmente, os indivíduos com maior atividade intelectual são os que possuem melhor desempenho cognitivo e, nesse sentido, esta avaliação poderia ser um melhor índice para a separação de grupos do que o número de anos de educação formal.

Os resultados deste estudo revelam a importância da manutenção do relacionamento social e familiar frequentes como estímulo adicional para a integridade funcional cognitiva. De acordo com Caldas \& Guerreiro, ${ }^{29}$ isso se deve "possivelmente pela manutenção da densidade crítica de sinapses neocorticais". Silberman et al. ${ }^{16}$ sugerem que a manutenção de muitos contatos e atividades sociais pode ajudar a prevenir ou adiar o declínio cognitivo em idosos, e mostraram em seu estudo escore bem baixo para o grupo de idosos que não tinham nenhuma relação social.
Quanto à influência de doenças crônicas, os resultados deste estudo estão em concordância com Veras, ${ }^{30}$ que relatou a existência de um risco relativo entre má saúde e deficiência cognitiva. Fratiglioni et al. ${ }^{31}$ verificaram que a possível causa do baixo escore no MEEM pode ser a presença de doenças somáticas, tais como cardiovasculares, diabetes e doenças respiratórias.

Os resultados deste estudo mostraram associação com depressão, que interferiu no teste de função cognitiva. No estudo de Raji et al., ${ }^{32}$ concluiu-se que um bom estado emocional minimiza o impacto na função cognitiva e física. O mesmo resultado foi obtido por Paternit et al. ${ }^{33}$

\section{CONCLUSÃO}

O presente estudo concluiu que o desempenho cognitivo dos idosos avaliados revelou que 18,3\% apresentaram escores abaixo do ponto de corte estabelecido (menor ou igual a 23).

Em relação a outras variáveis, o estudo mostrou que o desempenho no teste cognitivo foi melhor na faixa etária mais jovem, no nível melhor de escolaridade, entre aqueles com hábito de leituras frequentes e os que tinham suporte familiar frequente. Doenças crônicas como hipertensão arterial, diabetes, catarata, incontinência urinária e estado depressivo também influenciaram negativamente no desempenho do teste.

É de grande importância reconhecer possíveis casos de déficit cognitivo, que pode ser um precursor de demência com consequências para o indivíduo, a família e a sociedade. A partir desses dados, novas pesquisas poderão ser realizadas através de baterias neuropsicológicas com os idosos que ficaram abaixo do ponto de corte.

O envelhecimento populacional faz parte da realidade brasileira, e os idosos devem ser vistos como seus cidadãos, com direito ao envelhecer sadio, que é meta desejável de qualquer sociedade em desenvolvimento. A velhice deve 
ser acompanhada por melhorias na qualidade de vida através da promoção da saúde, ao lado do tratamento das doenças, com a criação de um ambiente social sadio e com o aumento dos cuidados consigo próprios.

\section{REFERÊNCIAS}

1. Silvestre JA, Kalache A, Ramos LR, Veras RP. O envelhecimento populacional brasileiro e o setor saúde. Arquivos de geriatria e gerontologia 1996; 0(1):81-9.

2. Ramos LR, Rosa TEC, Oliveira ZM, Medina MCG, Santos FRG. Perfil do idoso em área metropolitana na região sudeste do Brasil: resultados de inquérito domiciliar. Rev Saude Publica. 1993; 27(2):87-94.

3. Laks J, Rozenghal M, Engelhardt E. Sintomas psiquiátricos na doença de Alzheimer e sua relação com o estado cognitivo. Revista brasileira de neurologia e psiquiatria. 1995; 31(5):225-34.

4. Ramos LR, Macedo MB. Como diagnosticar e tratar: distúrbios de memória e demência. Rev Bras Méd. 2000; 57(12):87-92.

5. Geerlings MI, Jonker C, Bouter LM, Ader HJ, Schmand B. Associations between memory complaints and incident Alzheimer disease in elderly people with normal baseline cognition. Am J Psychiatry. 1999; 156(4):531-7.

6. Bickel H, Cooper B. Incidence and relative risk of dementia in an urban elderly population: findings of a prospective field study. Psychol Méd. 1994; 24:179-92.

7. Engelhardt E, Laks J, Rozenthal M, Von Poser NAS, Menkes C, Franco Neto CPB et al. Idosos velhos ("Oldest old"), rastreamento cognitivo com o MMSE. Revista brasileira de neurologia e psiquiatria. 1997; 33(4):201-6.

8. IBGE. Instituto Brasileiro de Geografia e Estatística. http://www.ibge.gov.br (acessado em 15 jul 2002).

9. Kish L. Survey Sampling. New York: John Wiley \& Sons, 1965.

10. Almeida OP, Almeida AS. Confiabilidade da versão brasileira da Escala de Depressão em Geriatria (GDS). Versão reduzida. Arq Neuropsiquiatr. 1999; 57(2-B):421-6.

11. Folstein MF, Folstein SE, Mchugh PR. "Mini-mental state": a practical method for grading the cognitive state of patients for the clinician. J Psychiatr. Res1975; 12:189-98.
É importante ressaltar que o cuidado de saúde destinado ao idoso é bastante caro, e a pesquisa corretamente orientada pode propiciar os instrumentos mais adequados para uma melhor definição de prioridades e alocação de recursos.

12. Seabra MLV, Concílio GV, Villares JB, Carlini EA. Avaliação do teste "Mini-Mental State" em voluntários e pacientes brasileiros. Revista brasileira de psiquiatria. 1990; 12(1-4):1-7.

13. Kleibaum DG. Logistic regression: a self-learning text. New York: Springer-Verlag; 1992.

14. Bennett DA, Wilson RS, Schneider JA, Evans DA, Beckett LA, Aggarwal NT, et al. Natural history of mild cognitive impairment in older persons. Neurology. 2002; 59:198-205.

15. Fichter MM, Meller I, Schröpell H, Steinkirchner R. Dementia and cognitive impairment in the oldest old in the community. Prevalence and comorbidity. $\mathrm{Br} J$ Psychiatry. 1995; 166:621-9.

16. Silberman C, Souza C, Wilhems F, Kipper L, Wu V, Diogo C et al. Cognitive deficit and depressive symptoms in a community group of elderly people. A preliminary study. Rev Saude Publica. 1995; 29(6):444-50.

17. Machado JC, Ribeiro RCL, Leal PFG, Cotta RMM. Avaliação do declínio cognitivo e sua relação com as características socioeconômicas dos idosos em ViçosaMG. Rev Bras Epidemiol. 2007; 10(4):592-605.

18. Diniz BSO, Volpe FM, Tavares AR. Nível educacional e idade no desempenho no Miniexame do Estado Mental em idosos residentes na comunidade. Revista de psiquiatria clínica. 2007, 34 (1); 13-7.

19. Nguyen HT, Black SA, Ray LA, Espino DV, Markides KS. Predictors of decline in MMSE scores among older Mexican. J Gerontol A Biol Sci Med Sci. 2002; 57(3):181-5.

20. Okuno J, Yanagi H, Tomura S. Is cognitive imapairment a risk factor for poor compliance among in the community. Eur J Clin Pharmacol. 2001; 57(8):589-94.

21. Jones RN, Gallo JJ. Education bias in the mini mental state examination. Int Psychogeriatr. 2001; 13(3): 299-310.

22. Laks J, Vega U, Silberman C, Rozenthal M, Nigri FN, Freitas RC et al. Rastreamento Cognitivo em 
idosos esquizofrênico institucionalizados. Revista brasileira de psiquiatria. 2000; 22(4):159-63.

23. Germano Neto J. Queixas de Memória e desempenho mnemônico e cognitivo entre idosos [tese]. Ribeirão Preto: Faculdade de Medicina de Ribeirão Preto, Universidade de São Paulo; 1997.

24. Ritchie K, Touchon J, Ledésert B, Leibovici D, Gorce AMD. Establishing the limits and characteristics of normal age-related cognitive decline. Rev Epidemiol Sante Publique. 1997; 45:373-81.

25. Tangalos EG, Smith GE, Ivnik RJ, Petersen RC, Kokmen E, Kurland LT, et al. The Mini Mental State Examination in general Medical Practice: clinical Utility and acceptance. Mayo Clin Proc. 1996; 71(9):829-37.

26. Barker A, Jones R, Jennison C. A prevalence study of age-associated memory impairment. Br J Psychiatry. 1995; 167:642-8.

27. Bertolucci PHF, Brucki SMD, Campacci SR, Juliano Y. O mini exame do estado mental em uma população geral. Arq Neuropsiquiatr.1994; 52(1):1-7.

28. Breteler MMB, Claus JJ, Grobbee DE, Hofman A. Cardiovascular disease and distribuition of cognitive function in elderly people: the Rotterdam Study. BMJ. 1994; 308:1604-8.

29. Caldas CP, Guerreiro TE. Memória e Demência (re) conhecimento e cuidado. Rio de Janeiro: UERJ/ UNATI; 2001.

30. Veras RP. País jovem com cabelos brancos: a saúde do idoso no Brasil. Rio de Janeiro: Relume Dumará/ UERJ; 1994.

31. Fratiglioni L, Viitanen M, Willard B. Very old women at highest risk of dementia and Alzheimer disease: incidence data from the Kungsholmenn Project Stockholm. Neurology. 1997; 48:132-8.

32. Raji MA, Ostir GV, Marvides KS, Goodwin JS. The interaction of cognitive and emotional status on subsequent physical functioning in older mexican american's finding from the hispanic estblished population for the epidemiologic study of the elderly. J Gerontol A Biol Sci Med Sci. 2002; 57(10):M678-82.

33. Paternit S, Verdier-Taillerfer MH, Dufouil C, Alperovitch A. Depression syntoms and cognitive decline in elderly people. Longitudinal study. Br J Psichiatry. 2002; 181:406-10. 